\title{
Experimental and Kinetic Modeling of the Ignition Delays of Cyclohexane, Cyclohexene, and Cyclohexadienes: Effect of unsaturation
}

L. Giarracca ${ }^{1,2}$, F. Isufaj ${ }^{1}$, J.-C. Lizardo-Huerta ${ }^{1}$, R. Fournet ${ }^{1}$, P.-A. Glaude ${ }^{1}$, B. Sirjean ${ }^{1, *}$

${ }^{1}$ Laboratoire Réactions et Génie des Procédés, CNRS, Université de Lorraine 1 rue Grandville BP 2045154001 Nancy Cedex, France

${ }^{2}$ IFP Energies Nouvelles, 1 et 4 avenue de Bois-Préau, 92852 Rueil-Malmaison Cedex, France

Corresponding author :

Baptiste Sirjean

Laboratoire Réactions et Génie des Procédés

1 rue Grandville BP 2045154001 Nancy Cedex,

France

Email: baptiste.sirjean@univ-lorraine.fr

Word count:

Main text: 3036 words

Figure $1(81+10) * 2.2+20=195$

Figure $2(46+10) * 2.2+25=150$

Figure $3(66+10) * 2.2 * 2+22=356$

Figure $4(51+10) * 2.2+31=165$

Figure $5(58+10) * 2.2+25=175$

Figure $6(51+10) * 2.2+32=166$

Figure $7(57+10) * 2.2+35=185$

Figure $8(57+10) * 2.2+35=181$

Figure $9(57+10) * 2.2+35=185$

Figure $10(57+10) * 2.2+35=185$

Figure $11(120+10) * 2.2 * 2+35=607$

References $(20+2) * 2.3 * 7.6=385$

Total: 5790 


\begin{abstract}
Cyclic and aromatic hydrocarbons are important components of usual commercial fuels, with $\mathrm{C}_{6}$-rings being among the most abundant cyclic structures. The combustion chemistry of $\mathrm{C}_{6^{-}}$ rings involves different levels of unsaturation, either as reactive (aromatics, naphtenes, ...) or during through the intermediates formed during their combustion. In this work the ignition delays of cyclohexane, cyclohexene, 1,3-cyclohexadiene and 1,4-cyclohexadiene are systematically studied using experiments and kinetic modeling. Shock tube experiments were performed at high-temperature (above 1200K) and for mean pressures of 6 atm. A detailed chemical kinetic model was developed that includes the combustion chemistry of the four cyclo- $\mathrm{C}_{6}$ fuels. Electronic structure calculations were performed at the CCSD(T)/CBS//B2PLYP-D3 level of theory on the pericyclic reactions of the unsaturated fuels. Pressure-dependent rate coefficients were computed by solving the master equation, and include in the mechanism. The model was validated against the new ignition data and against data of the literature. It was able to reproduce the experimental ranking of reactivity: cyclohexene > 14-CHD > cyclohexane > benzene $\approx 13-$ CHD. Kinetic analyses were performed to explain this difference of reactivity. It is shown that pericyclic reactions play a major role in the initial decomposition of the unsaturated fuels.
\end{abstract}




\section{Introduction}

With the depletion of conventional oil fields, future transportation fuels (gasoline, diesel and jet) are expected to contain an increasing portion of cyclic compounds from unconventional oil sources such as oil sands, oil shale, and coal liquefaction oils [1]. Cyclic and aromatic hydrocarbons are also used as additives to improve the resistance to auto-ignition for modern spark-ignition engines [2]. Understanding the combustion chemistry of these compounds is therefore of considerable scientific and practical importance.

These six-carbon rings compounds (benzene, cyclohexadienes, cyclohexene and cyclohexane and their derivatives) are the among most abundant cyclic structures in commercial fuels. The different chemical structure, and in particular, their different level of unsaturation can affect their ignition behavior. The studies on the autoignition of these compounds are focused on benzene $[3,4]$, as the simplest aromatic species used as octane booster, and on cyclohexane [5-7] as the simple model of naphetenes class.

Among the different study on autoignition of cyclohexane, Sirjean et al. [7] measured the ignition delays for $\mathrm{C}_{6} \mathrm{H}_{12}-\mathrm{O}_{2}$ - $\mathrm{Ar}$ mixtures in shock tube at $1230-1840 \mathrm{~K}, 7.3-9.5 \mathrm{~atm}$, and equivalence ratios of $0.5,1$ and 2 . The results have shown that auto-ignition delays of cyclohexane are 10 times higher than that of cyclopentane for a given temperature. Hong et al [7] reported the ignition delay times for cyclohexane at 1.5 and 3 atm, equivalence ratios near 1 and 0.5 , and temperatures between 1280 and $1480 \mathrm{~K}$. Daley et al. [6] reported the ignition delay for cyclohexane/air mixture at lower temperature $(847-1379 \mathrm{~K})$ but higher pressure (11-61 atm) and equivalence ratios of $0.25,0.5$ and 1.0.

In the early 2000s, cyclohexene autoignition was studied in a low temperature range (600-900 $\mathrm{K}$ ) in a rapid compression machine [8] and at high temperatures in a shock tube by Dayma et al. [9]. The latter study measured the ignition delays of cyclohexene-oxygen-argon mixtures for 1 or $2 \%$ concentration of hydrocarbons and equivalence ratios ranging from 0.5 to 2 . Reflected shock waves permitted to obtain temperatures from 1050 to $1520 \mathrm{~K}$ and pressures 
from 7.7 to 9.1 atm. A detailed kinetic mechanism was also proposed, which was able to reproduce ignition delays.

Cyclohexadienes are important precursors of benzene. The low temperature oxidation of 1,3cyclohexadiene (13-CHD) was studied by Lemaire et al. [10] in a rapid compression machine and is the only experimental study on the combustion of these compounds available in the literature. Recently, Schönborn et al. [11] have published a detailed chemical kinetic model for the low-temperature (500-1200 K) combustion of 1,3-cyclohexadiene based on theoretical calculations for thermochemistry. Currently, there is no data available for the oxidation of cyclohexadienes $(1,3$ or 1,4$)$ at high-temperature conditions, although the chemistry of these compounds can be important for the formation of soot precursors from cyclohexane and its derivatives by dehydrogenation.

The aim of this present investigation is to compare the different ignition delay times of cyclohexane, cyclohexene and 1,3-and 1,4-cyclohexadiene (14-CHD) to understand the effect of different levels of molecular unsaturation. In 1979, Burcat et al. [12] compared the ignition delay times for selected ring-structured hydrocarbons, but, to the best of our knowledge, no study proposed a systematic comparison on the unsaturation effect on the same numbercarbon-ring structure.

In this work, a detailed kinetic model for the oxidation of cyclohexane, cyclohexene and 1,3and 1,4-cyclohexadiene are presented. This model is validated with data of ignition delay times measured in a shock tube, using the same conditions all these $\mathrm{C}_{6}$ rings compounds. The kinetics of key elementary reactions of unsaturated $\mathrm{C}_{6}$ of the mechanism are calculated using quantum chemistry, statistical thermodynamics and reaction rate theories.

\section{Experimental methods}

Ignition delay times of the fuels were measured in a shock tube which has already been described in details elsewhere [13]. Only the main features of the stainless-steel shock tube 
are given here. The driver (filled with helium) and reaction parts are separated by two Terphane diaphragms which were ruptured by a sudden drop of the pressure between them. Four piezo-electric pressure transducers are placed along the driver section to measure the incident and reflected shock waves velocities. Ideal one-dimensional shock equations are used to calculate the temperature and pressure of the fuel/ $\mathrm{O}_{2}$ mixture behind the reflected shock wave. The onset of ignition was detected by excited $\mathrm{OH}^{*}$ radical emission at $306 \mathrm{~nm}$ through a quartz window embedded in the sidewall with a photomultiplier fitted with a monochromator at the end of the reaction part (the time resolution of the emission measurements is better than $2 \mu \mathrm{s})$. The quartz window was located at the same place along the axis of the tube as the last pressure transducer. The ignition delay time was defined as the time interval between the pressure rise measured by the last pressure transducer due to the arrival of the reflected shock wave, and the rise of the optical signal by the photomultiplier up to $10 \%$ of its maximum value. A typical experimental profile, that includes the definition of ignition delay, is given in Figure S1. Uncertainty of temperatures behind the reflected shock wave $\left(T_{5}\right)$ is about $\pm 20 \mathrm{~K}$.

Cyclohexane was purchased from Biosolve, with a purity of 99.8\%, cyclohexene and 1,3 / 1,4-cyclohexadienes from Sigma-Aldrich with purities of 99\%, 97\% an 97\%, respectively. Oxygen, argon and helium were purchased from Messer (purity > 99.995). Fresh reaction mixtures were prepared every day and mixed using a recirculation pump. The reaction section was flushed with pure argon and evacuated before each fuel mixture testing.

\section{Kinetic model development}

A detailed kinetic model was developed for the combustion of cyclohexane, cyclohexene, 1,3and 1,4-cyclohexadienes to simulate experimental ignition delays. Since pericyclic reactions play a key role in the combustion chemistry of unsaturated cyclic $\mathrm{C}_{6}$ fuels, their thermokinetic parameters were computed using theoretical chemical kinetic tools. 


\subsection{Computational methods}

Potential energy surfaces of the pericyclic reactions of cyclohexene, 1,3-CHD and 1,4-CHD are computed using a dual-level approach. Geometry optimization and frequency calculations were performed at the B2PLYP-D3/cc-pVTZ level of theory. Single-point calculations were done at the $\operatorname{CSD}(\mathrm{T})$ level of theory with Complete Basis Set (CBS) extrapolation using ccpVQZ and cc-pVTZ basis sets, and the formula proposed by Halkier et al. [14]. The Gaussian09 code was used for all electronic structure calculations. Vibrational partition functions of cyclic molecular structures were computed within the harmonic oscillator approximation. In the case of 1,3,5-hexatriene, the partition function was corrected for internal rotors, using the 1D-HR method, based on relaxed scans performed at the B2PLYPD3/cc-pVDZ level. Rate coefficients were calculated using the MESS code to solve the Master Equation (ME). The collisional energy transfer probability was described by the exponential down model, with $\langle$ Edown $\rangle=200 \times(\mathrm{T} / 300)^{0.85}\left(\mathrm{~cm}^{-1}\right)$ for argon. Quantum tunneling was considered using the Eckart approach for reactions involving $\mathrm{H}$-atom transfers.

\subsection{Kinetic model description}

The high-temperature kinetic model is made of three interconnected sub-mechanisms for cyclohexane, cyclohexene and 1,3- / 1,4-cyclohexadiene that are appended to a reaction base that describes the oxidation of $\mathrm{H}_{2}$ and $\mathrm{CO}$ and the high-temperature chemistry of $\mathrm{C}_{1}-\mathrm{C}_{4}$ hydrocarbons and benzene (named "core mechanism" in the text).

The starting core mechanism was taken from a previous study from our group [11]. Several reactions of benzene and cyclopentadiene sub-mechanism were updated based on recent studies of the literature. Pressure dependent rate constants for the reaction of benzene with Oatoms were taken from the work of Taatjes et al. [15].

Cyclohexane sub-mechanism. Unimolecular initiation of cyclohexane, yielding 1-hexene was taken from Sirjean et al. [16] calculations. Initial C-H bond fission, bimolecular initiations with $\mathrm{O}_{2}, \mathrm{H}$-abstractions with $\mathrm{H}$ - and $\mathrm{O}$ - atoms, $\mathrm{OH}, \mathrm{HO}_{2}$, and $\mathrm{CH}_{3}$ were taken from the 
JetSurf v2.0 model [17]. Pressure dependent rate constants for the isomerizations and $\beta$ scissions of cyclohexyl radical and its acyclic isomers were also taken from JetSurf v2.0 and are based on the experimental work of Tsang and co-workers [18]. 1-hexene high-temperature chemistry was taken from Sirjean et al. [5].

Cyclohexene sub-mechanism. Pressure-dependent rate coefficients for the Retro-Diels-Alder and $\mathrm{H}_{2}$-elimination reactions were computed in the present study. Initial $\mathrm{C}-\mathrm{H}$ bond fissions were written as the reverse termination reactions and bimolecular initiations were taken from the work of Dayma et al. [9]. The kinetic parameters of $\mathrm{H}$-abstractions reactions are based on correlations derived from theoretical calculations and were included for allylic and alkylic $\mathrm{H}$ atoms of cyclohexene (abstraction of vinylic $\mathrm{H}$-atoms was neglected). Isomerization and $\beta$ scissions of cyclohexenyl radicals are part of the cyclohexadienes sub-mechanism and their decomposition reactions and associated rate constants were determined using theoretical calculations (see below). $\mathrm{OH}$ and $\mathrm{O}$-atom additions on cyclohexene were also included as lumped reactions based on the reaction rate rules of the EXGAS kinetic model generator software [19].

Cyclohexadienes sub-mechanism. 13-CHD and 14-CHD pericyclic reactions are interconnected and their pressure-dependent rate coefficients were computed in this study. Initial $\mathrm{C}-\mathrm{H}$ bond fission were written as the reverse termination reactions with pressuredependence based on analogies with the phenyl $+\mathrm{H}$ reaction. The rate constants of bimolecular initiations with $\mathrm{O}_{2}, \mathrm{H}$-abstractions with $\mathrm{H}$ - and $\mathrm{O}$ - atoms, $\mathrm{OH}, \mathrm{HO}_{2}, \mathrm{CH}_{3}$, and C$\mathrm{H} \beta$-scission from cyclohexadienyl radical were taken from the recently published lowtemperature combustion kinetic model of 13-CHD [11]. O-atoms addition on 13-CHD and 14-CHD were written based on analogies with the work of Taatjes et al. on benzene $+O\left({ }^{3} \mathrm{P}\right)$. H-additions pathways were calculated at the CBS-QB3 level of theory. The computed potential energy surfaces (PES) are given in Figure S2. From these PESs, thermochemical data were calculated, including a 1D-HR-U treatment for internal rotations based on 
B3LYP/6-311G(d,p) relaxed scans. High-pressure limit rate constants were computed using transition state theory and the rigid rotor (1D-HR-U) harmonic oscillator approximation, with Eckart tunneling. The ThermRot software [20] was used for the calculation of these data from Gaussian09 calculation results. 1,3,5-hexatriene (135-HT) is a major intermediate in 13-CHD combustion chemistry [11]. Its combustion reactions were included in the kinetic model with bimolecular initiations with $\mathrm{O} 2$ and $\mathrm{H}$-abstractions taken from the low-temperature mechanism of 13-CHD [11]. Initial C-H bond fissions were added in the present work based on the reverse termination. The isomerization and $\beta$-scission reactions of hexatrienyl radicals were computed in this study, using the same methodology as in the case of H-additions onto 13- and 14- CHD. O-addition were written based on analogies with 1,3-butadiene $+\mathrm{O}\left({ }^{3} \mathrm{P}\right)$.

The new kinetic model for cyclohexane, cyclohexene and cyclohexadienes high temperature combustion $(\mathrm{T}>1100 \mathrm{~K})$ features 2318 reactions and 367 species.

\subsection{Theoretical results for pericyclic reactions}

In the cyclic- $\mathrm{C}_{6}$ hydrocarbon series, cyclohexene and 13- and 14-CHD can decompose through pericyclic reactions, while this type of reaction cannot occur for cyclohexane and benzene.

Figure 1 presents the computed potential energy surface for the classic retro-Diels-Alder reaction and $\mathrm{H}_{2}$-elimination of cyclohexene.

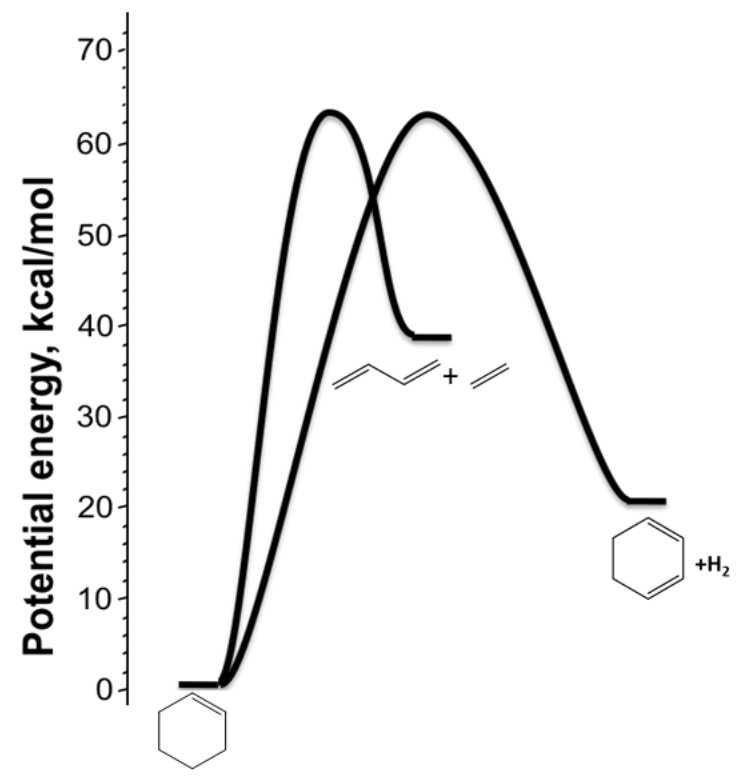


Figure 1: Potential energy surface of pericyclic decompositions of cyclohexene. Energy at 0K, computed at the CCSD(T)/CBS//B2PLYP-D3/cc-pvTZ level of theory.

The critical energy computed at the $\operatorname{CCSD}(\mathrm{T}) / \mathrm{CBS}$ level of calculation for the retro-DielsAlder reaction is $63.1 \mathrm{kcal} / \mathrm{mol} . \mathrm{H}_{2}$-elimination faces a slightly lower energy barrier of 61.9 $\mathrm{kcal} / \mathrm{mol}$.

Figure 2 depicts the computed rate coefficients for the two pericyclic reactions of cyclohexene, compared to experimental data of the literature.

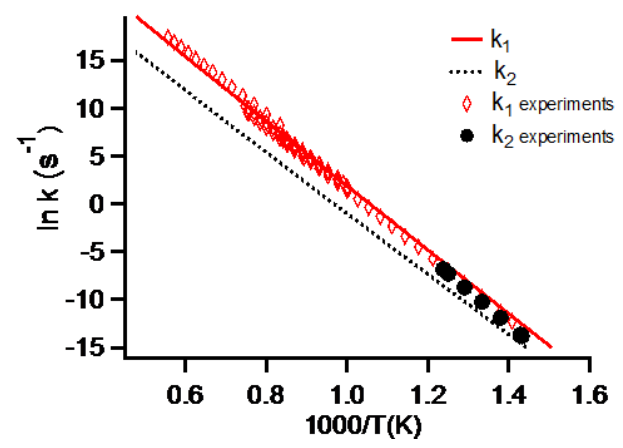

Figure 2: Comparisons between experimental (retrieved from NIST kinetics database) and calculated rate constants for the pericyclic reactions of cyclohexene. (1) retro-DielsAlder reaction; (2) $\mathrm{H}_{2}$-elimination.

The computed rate constants are in agreement with the experimental data, given the scatter of the different measurements. The mean deviation for the retro-Diels Alder reaction is $39.8 \%$. Above $1000 \mathrm{~K}$, the retro-Diels Alder reaction is favored over $\mathrm{H}_{2}$-elimination. The pressure dependence of the rate constants is presented in Figure S3. Below $1200 \mathrm{~K}$, almost no fall-off is observed. For the retro-Diels-Alder reaction, a factor of 109 is computed at $2000 \mathrm{~K}$ for $\mathrm{k}_{\mathrm{inf}} / \mathrm{k}_{0.1 \text { bar. }}$ At the same temperature, for 10 bar, a factor of 5 is observed.

Figure 3 depicts the potential energy surface for 13-CHD and 14-CHD pericyclic reactions 


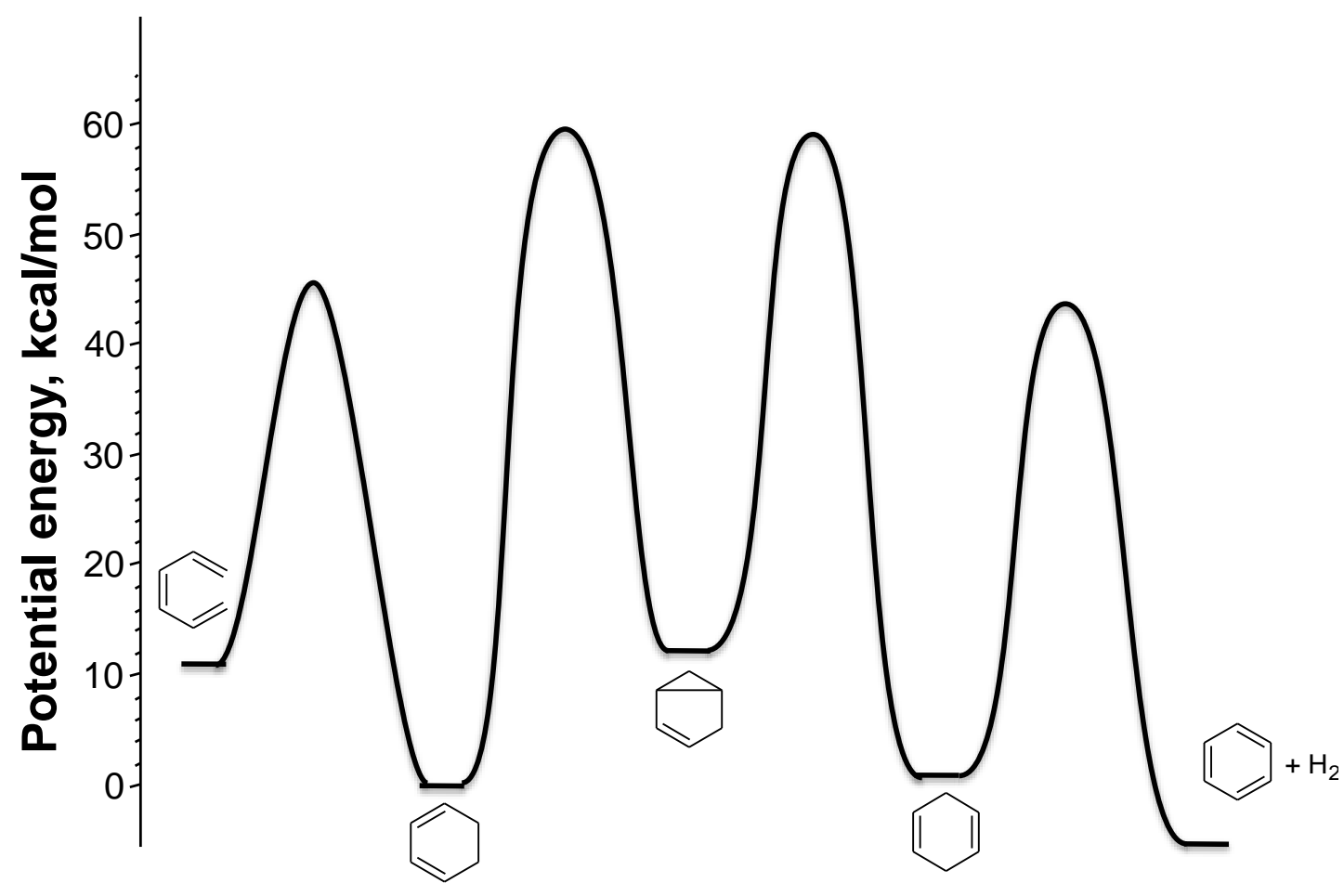

Figure 3: Potential energy surface of pericyclic decompositions of 13- and 14-CHD. Energy at 0K, computed at the CCSD(T)/CBS//B2PLYP-D3/cc-pvTZ level of theory.

13-CHD and 14-CHD are connected through the formation of bicyclohexene via energy barriers of 60.5 and $61.1 \mathrm{kcal} / \mathrm{mol}$, respectively. The cyclization of cis-1,3,5-hexatriene into 13-CHD faces an energy barrier of $30.7 \mathrm{kcal} / \mathrm{mol} . \mathrm{H}_{2}$-elimination in 14-CHD involves a critical energy of $45.4 \mathrm{kcal} / \mathrm{mol}$. Figure 4 presents a comparison between the computed and experimental kinetic data of the literature.

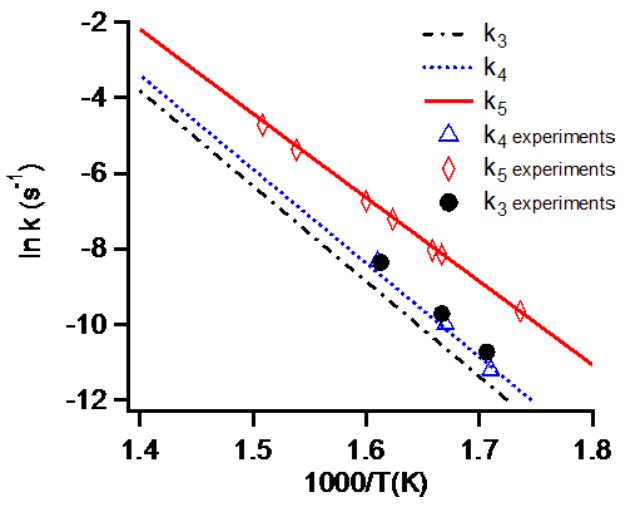

Figure 4: Comparisons between experimental (retrieved from NIST kinetics database) and calculated rate constants for the pericyclic reactions of 13- and 14-CHD. (3) bicyclohexene $\rightarrow$ 14-CHD, (4) bicyclohexene $\rightarrow$ 13-CHD, (5) 14-CHD $\rightarrow$ benzene $+\mathrm{H}_{2}$ 
The computed high-pressure rate constants agree with the limited experimental data of the literature. The largest deviation is observed for bicyclohexene isomerization into 14-CHD (reaction (3), while the rate constant for $\mathrm{H}_{2}$-elimination in 14-CHD shows a maximum deviation of $11.9 \%$. Rate coefficients at different pressures are available in the kinetic model in the supplemental material. Fall-off effects are more pronounced for 13-CHD and 14-CHD rearrangements into bicyclohexene and for 13-CHD into 135-HT, respectively. At $1500 \mathrm{~K}$ and 1 atm, the ratios of $k_{\text {inf }} / k_{1}$ bar are 4 for reaction (3), 3 for reaction (4) while the pressure dependence is small for $\mathrm{H}_{2}$-elimination in 14-CHD (see Figure S3).

\section{Experimental and simulation results}

Simulations of ignition delay times of cyclic $\mathrm{C}_{6}$-fuels/ $\mathrm{O}_{2} / \mathrm{Ar}$ mixtures were performed using an adiabatic homogeneous batch reactor with the Chemkin-Pro software. In the simulations, ignition delay time definition was taken as $10 \%$ of the peak of $\mathrm{OH}$, as in the experiments. Figure 5 presents a comparison between the experimental and simulated ignition delays for cyclohexane

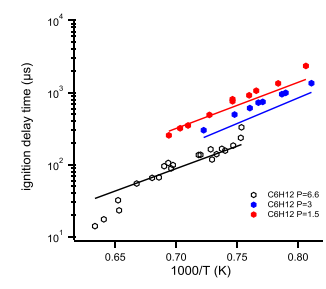

Figure 5: Experimental and simulated ignition delay times of cyclohexane $/ \mathrm{O}_{2} / \mathrm{Ar}$ mixtures at equivalence ratio of 1. Open symbols: experiments from present work with $0.5 \%$ of $\mathrm{C}_{6} \mathrm{H}_{12}$ at $\mathrm{P}_{5}=6.6$ atm. Filled symbols: Hong et al. [7] data for $0.4 \%$ of fuel at 3 and $1.5 \mathrm{~atm}$. Lines: simulations.

Since the literature data in shock tube are abundant, new data for cyclohexane were measured only at one condition that was chosen to be identical to other cyclic $\mathrm{C}_{6}$ fuels studied. The model is able to capture correctly the behavior of cyclohexane ignition delays at different 
pressures. Simulations underestimate experimental data at 3 atm, with a mean deviation of $30 \%$.

Figure 6 depicts the ignition delays of cyclohexene.

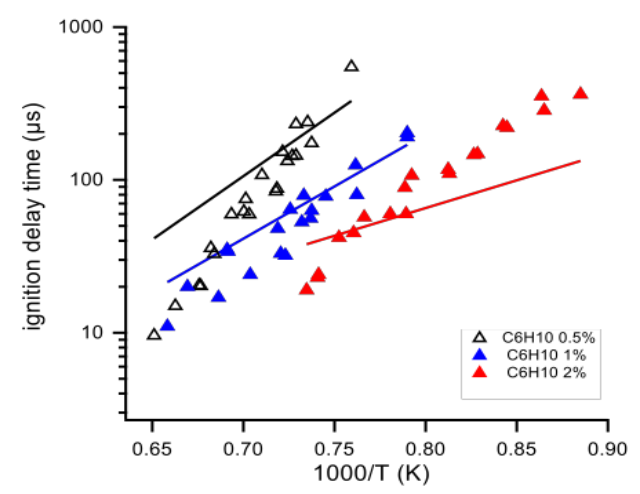

Figure 6: Experimental and simulated ignition delay times of cyclohexene/ $/ \mathrm{O}_{2} / \mathrm{Ar}$ mixtures at equivalence ratio of 1 and different fuel concentrations. Open symbols: experiments from present work with $0.5 \%$ of $\mathrm{C}_{6} \mathrm{H}_{10}$ at $\mathrm{P}_{5}=6.4 \mathrm{~atm}$. Filled symbols: Dayma et al. [9] data for two different fuel concentrations at 8.5 atm. Lines: simulations.

Cyclohexane data were measured in this study for one condition because other data for cyclohexene ignition were measured in our shock tube in a previous study from Dayma et al.

[9]. Overall, the kinetic model agrees with the experimental data but an underestimation of simulated ignition delay is observed for $2 \%$ fuel concentration at the lowest temperatures.

Figure 7 presents the ignition delays of 1,3-CHD.

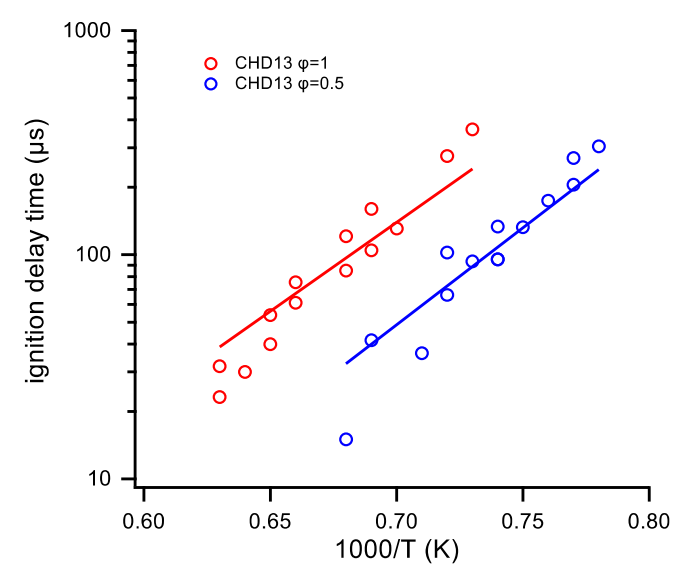

Figure 7: Experimental and simulated ignition delay times of $13-\mathrm{CHD} / \mathrm{O}_{2} / \mathrm{Ar}$ mixtures at equivalence ratio of 1 and 0.5 . Open symbols: experiments from present work with $0.5 \%$ of $13-\mathrm{CHD}$ at $\mathrm{P}_{5}=6.5$ atm. Lines: simulations. 
The model is able to capture the ignition behavior of 13-CHD at two different equivalence ratios. To the best of our knowledge, ignition delays presented in Figure 7 are the first of the literature at high temperature.

Figure 8 presents the ignition delays of 14-CHD.

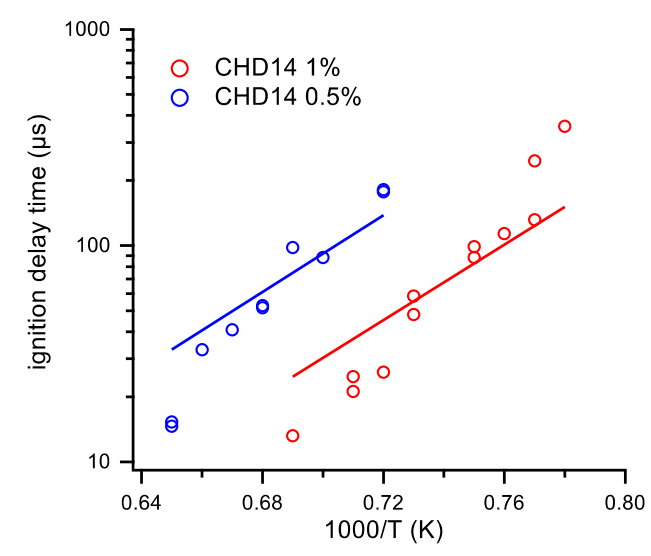

Figure 8: Experimental and simulated ignition delay times of $14-\mathrm{CHD} / \mathrm{O}_{2} / \mathrm{Ar}$ mixtures at equivalence ratio of 1 and 0.5. Open symbols: experiments from present work with $0.5 \%$ of $14-\mathrm{CHD}$ at $P_{5}=6.5 \mathrm{~atm}$. Lines: simulations.

It appears that under the same conditions, ignition delay times of 14-CHD are shorter than of 13-CHD despite the similarity in the chemical structure. The model is able to simulate this observed behavior. Figure 9 presents a comparison between 13- and 14-CHD ignition delays.

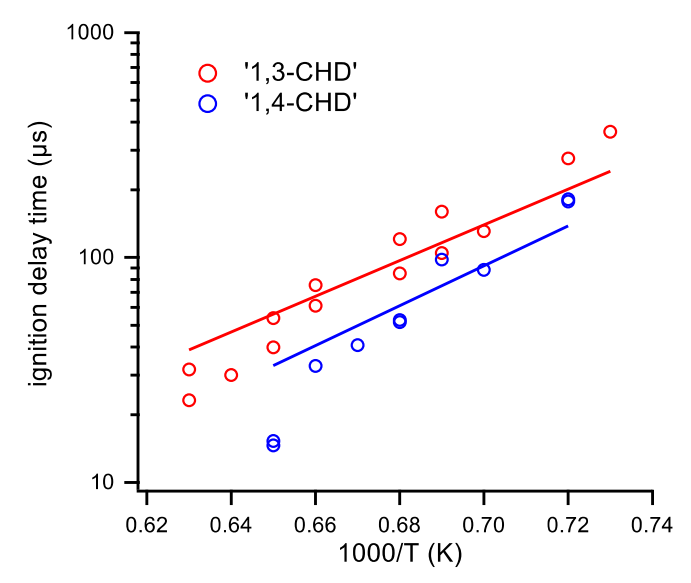

Figure 9: Experimental and simulated ignition delay times of 14- and 13-CHD/O $/ \mathrm{O}_{2} / \mathrm{Ar}$ mixtures at equivalence ratio of 1 . Open symbols: experiments from present work with $0.5 \%$ of $14-\mathrm{CHD}$ at $\mathrm{P}_{5}=6.5$ atm. Lines: simulations.

Kinetic analyses presented at the end of the manuscript will highlight the reasons of the difference of reactivity between these two fuels. 
Figure 10 present a comparison between the ignition delay times of cyclohexane, cyclohexene, 13- and 14-CHD and a new set of data for benzene. All data were measured in the same conditions.

Figure 10: Experimental ignition delay times of cyclohexane (pink squares), cyclohexene (green triangles), 14- (blue circles), 13-CHD (red circles) and benzene (black circles) $/ \mathrm{O}_{2} / \mathrm{Ar}$ mixtures at equivalence ratio of 1, with $0.5 \%$ fuel concentration at $P_{5}=6.5 \mathrm{~atm}$.

It is observed that benzene and 13-CHD ignition delays are similar and that they are the higher values among the four fuels. Ignition delays of cyclohexene are the shortest and are close to those of cyclohexane below $1400 \mathrm{~K}$. Above this temperature, ignition delays of cyclohexane are longer and are similar to those of 14-CHD. Above $1400 \mathrm{~K}$, the ranking of reactivity is cyclohexene $>14-\mathrm{CHD}>$ cyclohexane $>$ benzene $\approx 13-\mathrm{CHD}$.

Figure 11 presents a reaction flux analysis performed with the kinetic model for the four different fuels.

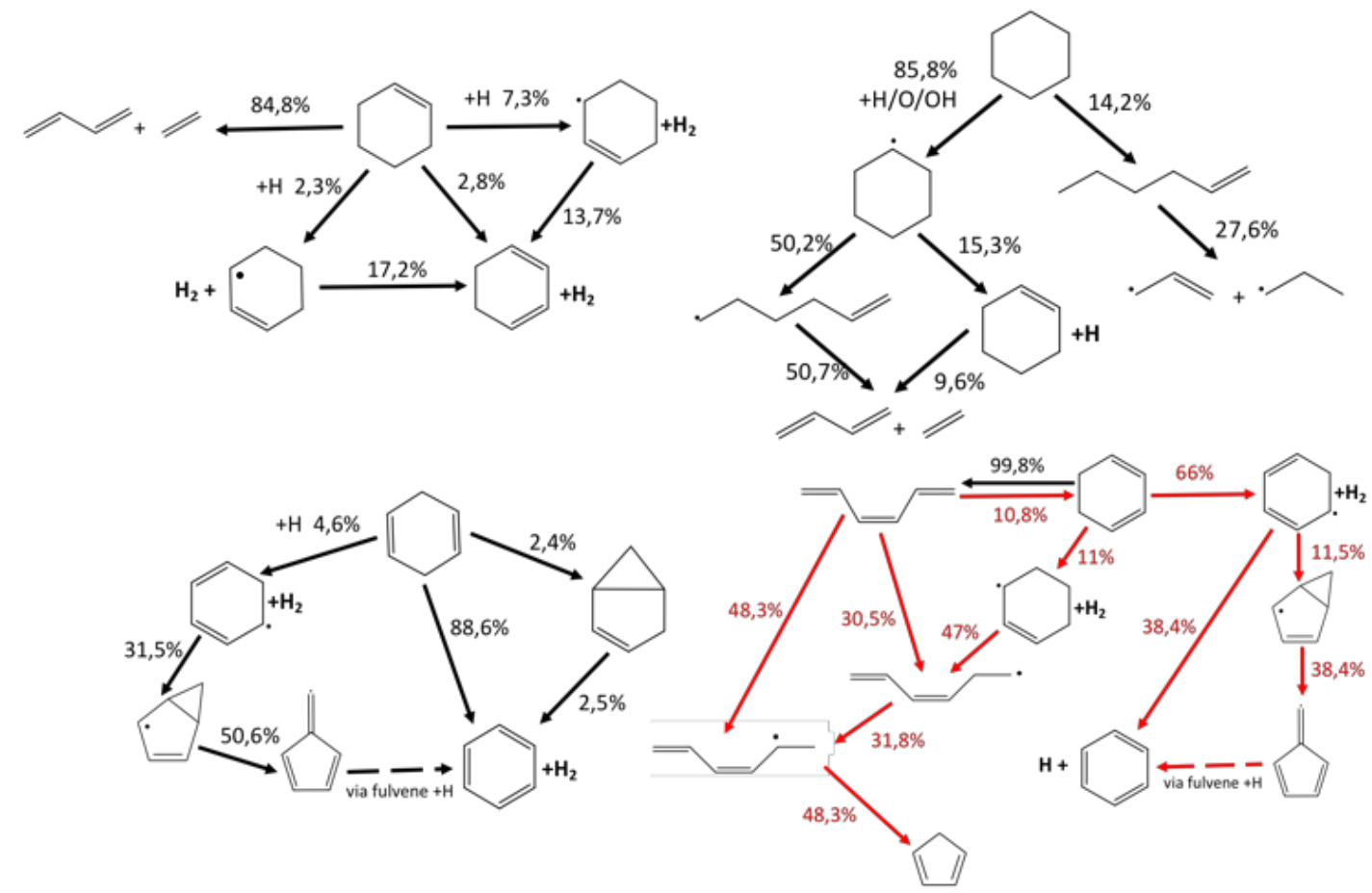

Figure 11: Reaction flux analysis for cyclohexane (top right), cyclohexene (top left), 14-CHD (bottom left) and 13-CHD (bottom right). Simulations performed at $1400 \mathrm{~K}$ and $6.5 \mathrm{~atm}$. Arrows represent the total flux in percent, relative to the initial fuel consumption. Black: 10\% fuel conversion. Red: 50\% fuel conversion. 
Adding unsaturation to cyclohexane leads to an increased role of pericyclic reactions. For cyclohexene, the retro-Diels-Alder reaction represents $84.8 \%$ of the fuel consumption. $\mathrm{H}_{2-}$ elimination constitute $88.6 \%$ of 14-CHD consumption and 1,3,5-hexatriene formation represents $99.8 \%$ of $13-\mathrm{CHD}$ consumption at $10 \%$ conversion. The initial consumption flux of cyclohexane mainly involves $\mathrm{H}$-abstraction reactions, followed by the unimolecular decomposition of cyclohexyl radicals. Under the condition studied, $15 \%$ of the total cyclohexane consumption leads to the formation of cyclohexene. Almost $15 \%$ of the decomposition flux of cyclohexene yields 13-CHD. 14- and 13-CHD lead to the formation of benzene. The difference of reactivity between the fuels can be explained by the observed fluxes. 13-CHD features the lowest reactivity because of its easy formation of 135-HT which inhibits the reactivity. 14-CHD majorly yields benzene $+\mathrm{H}_{2}$, but also feature consumption fluxes leading to the formation of cyclohexadienyl radical by $\mathrm{H}$-abstractions. The latter radical is involved in chain reactions that creates free radicals that are involved in reactions that promotes reactivity (see brute force sensitivity analyses in Figure S4). The difference of reactivity between cyclohexane and cyclohexene can be explained by the favorable initial C$\mathrm{H}$ bond fission in cyclohexene that creates reactive $\mathrm{H}$-radicals while the unimolecular initiation of cyclohexane yields first yields 1-hexene that further decomposes into the inhibiting allyl radical and propyl radical. Brute force sensitivity analyses are presented in Figure S4.

\section{Conclusions}

In this study, the effect of different levels of unsaturation on cyclo- $\mathrm{C}_{6}$ species on hightemperature $(>1200 \mathrm{~K})$ ignition delay times was explored using shock tube experiments and kinetic modelling. Ignition delays behind reflected shock tubes were measured under the same conditions $(0.5 \%$ fuel concentration, stoichiometric equivalence ratio, and a mean pressure of $6.5 \mathrm{~atm})$. Experimental data showed that the ranking of reactivity at these conditions is cyclohexene $>14-$ CHD $>$ cyclohexane $>$ benzene $\approx 13-$ CHD, above $1400 \mathrm{~K}$. Ignition data for 
1,3-cyclohexadiene and 1,4-cyclohexadiene are the first ones reported in the literature. A detailed kinetic model was also developed to simulate the experimental data. High-level quantum calculations at the CCSD(T)/CBS//B2PLYP-D3/cc-pvTZ were performed on all the pericyclic decompositions of the unsaturated fuels. Master equation simulations were performed to determine pressure dependent rate coefficients of these reactions. The main effect of adding unsaturation on a $\mathrm{C}_{6}$ ring is to enable these pericyclic reactions. In cyclohexene, the retro-Diels-Alder reaction is found to be a major initial dissociation route. $\mathrm{H}_{2}$-elimination in 14-CHD was found to be the major initial decomposition that directly yields benzene. In 13-CHD, the formation 135-HT is preponderant at early conversion and inhibits the reactivity. The competition between the pericyclic reaction that leads to the formation of molecules that further creates the first radicals, and initial $\mathrm{C}-\mathrm{H}$ and $\mathrm{C}-\mathrm{C}$ bond fissions explain the difference of reactivity observed in experiments.

\section{Supplemental material}

The detailed chemical kinetic model is available as supplemental material. Pressuredependent rate coefficient plots and sensitivity analyses are also available.

\section{Acknowledgements}

High performance computing resources were partially provided by the EXPLOR center hosted by the University of Lorraine. This work was also granted access to the HPC resources of IDRIS under the allocation 2018-A0010807249 made by GENCI. F.R Mendy is acknowledged for his help with experiments. 


\section{References}

[1] Y. Yang, A.L. Boehman, Oxidation chemistry of cyclic hydrocarbons in a motored engine: Methylcyclopentane, tetralin, and decalin, Combustion and Flame 157 (2010) 495505 .

[2] M.D. Boot, M. Tian, E.J. Hensen, S.M. Sarathy, Impact of fuel molecular structure on auto-ignition behavior-Design rules for future high performance gasolines, Progress in Energy and Combustion Science 60 (2017) 1-25.

[3] I.D. Costa, R. Fournet, F. Billaud, F. Battin- Leclerc, Experimental and modeling study of the oxidation of benzene, International journal of chemical kinetics 35 (2003) 503-524.

[4] G. Mittal, C.-J. Sung, Autoignition of toluene and benzene at elevated pressures in a rapid compression machine, Combustion and Flame 150 (2007) 355-368.

[5] B. Sirjean, F. Buda, H. Hakka, P.-A. Glaude, R. Fournet, V. Warth, F. Battin-Leclerc, M. Ruiz-Lopez, The autoignition of cyclopentane and cyclohexane in a shock tube, Proceedings of the Combustion Institute 31 (2007) 277-284.

[6] S.M. Daley, A.M. Berkowitz, M.A. Oehlschlaeger, A shock tube study of cyclopentane and cyclohexane ignition at elevated pressures, International Journal of Chemical Kinetics 40 (2008) 624-634.

[7] Z. Hong, K.-Y. Lam, D.F. Davidson, R.K. Hanson, A comparative study of the oxidation characteristics of cyclohexane, methylcyclohexane, and n-butylcyclohexane at high temperatures, Combustion and Flame 158 (2011) 1456-1468.

[8] M. Ribaucour, O. Lemaire, R. Minetti, Low-temperature oxidation and autoignition of cyclohexene: A modeling study, Proceedings of the Combustion Institute 29 (2002) 13031310.

[9] G. Dayma, P. Glaude, R. Fournet, F. Battin- Leclerc, Experimental and modeling study of the oxidation of cyclohexene, International journal of chemical kinetics 35 (2003) 273-285.

[10] O. Lemaire, M. Ribaucour, M. Carlier, R. Minetti, The production of benzene in the lowtemperature oxidation of cyclohexane, cyclohexene, and cyclohexa-1, 3-diene, Combustion and flame 127 (2001) 1971-1980.

[11] A. Schönborn, M.D. Le, R. Fournet, P.-A. Glaude, V. Warth, B. Sirjean, Auto-ignition control using an additive with adaptable chemical structure. Part I: Development of a kinetic model for 1, 3-cyclohexadiene and 1, 3, 5-hexatriene combustion, Combustion and Flame 205 (2019) 466-483.

[12] A. Burcat, R. Farmer, R. Espinoza, R. Matula, Comparative ignition delay times for selected ring-structured hydrocarbons, Combustion and Flame 36 (1979) 313-316.

[13] R. Fournet, J. Bauge, F. Battin- Leclerc, Experimental and modeling of oxidation of acetylene, propyne, allene and 1,3- butadiene, International journal of chemical kinetics 31 (1999) 361-379.

[14] A. Halkier, T. Helgaker, P. Jørgensen, W. Klopper, H. Koch, J. Olsen, A.K. Wilson, Basis-set convergence in correlated calculations on $\mathrm{Ne}, \mathrm{N} 2$, and $\mathrm{H} 2 \mathrm{O}$, Chemical Physics Letters 286 (1998) 243-252.

[15] C.A. Taatjes, D.L. Osborn, T.M. Selby, G. Meloni, A.J. Trevitt, E. Epifanovsky, A.I. Krylov, B. Sirjean, E. Dames, H. Wang, Products of the benzene+ O (3P) reaction, The Journal of Physical Chemistry A 114 (2010) 3355-3370.

[16] B. Sirjean, P.-A. Glaude, M. Ruiz-Lopez, R. Fournet, Detailed kinetic study of the ring opening of cycloalkanes by CBS-QB3 calculations, The Journal of Physical Chemistry A 110 (2006) 12693-12704.

[17] H. Wang, E. Dames, B. Sirjean, D. Sheen, R. Tangko, A. Violi, J. Lai, F. Egolfopoulos, D. Davidson, R. Hanson, A hightemperature chemical kinetic model of n-alkane (up to ndodecane), cyclohexane, and methyl-, ethyl-, n-propyl and n-butyl-cyclohexane oxidation at high temperatures, JetSurF version 2.0; September 19, 2010, URL (http://melchior. usc. edu/JetSurF/JetSurF2. 0) (2010) 17-111. 
[18] I.A. Awan, W.S. McGivern, W. Tsang, J.A. Manion, Decomposition and isomerization of 5-methylhex-1-yl radical, The Journal of Physical Chemistry A 114 (2010) 7832-7846.

[19] F. Buda, R. Bounaceur, V. Warth, P.-A. Glaude, R. Fournet, F. Battin-Leclerc, Progress toward a unified detailed kinetic model for the autoignition of alkanes from $\mathrm{C} 4$ to $\mathrm{C} 10$ between 600 and $1200 \mathrm{~K}$, Combustion and flame 142 (2005) 170-186.

[20] J. Lizardo-Huerta, B. Sirjean, R. Bounaceur, R. Fournet, Intramolecular effects on the kinetics of unimolecular reactions of $\beta$-HOROO and $\mathrm{HOQ}^{\circ} \mathrm{OOH}$ radicals, Physical Chemistry Chemical Physics 18 (2016) 12231-12251. 